\title{
Reciprocal Negotiation Over Shared Resources in Agent Societies
}

\author{
Sabyasachi Saha \\ sabyasachi.saha@gmail.com \\ Sandip Sen \\ sandip@utulsa.edu \\ Mathematical \& Computer Sciences Department \\ University of Tulsa \\ Tulsa, Oklahoma, USA
}

\begin{abstract}
We are interested in domains where an agent repeatedly negotiates with other agents over shared resources where the demand or utility to the agent for the shared resources vary over time. We propose a protocol that will maximize social welfare if agents reveal their true preferences in every negotiation. The protocol, however, is not truth-revealing and selfish agents have the incentive to artificially inflate preferences. We use a probabilistic reciprocative behavior that discourages the reporting of false preferences. This reciprocative behavior promotes cooperation in repeated negotiations and improves both individual and group longterm payoff. We characterize environmental conditions under which agents can develop and sustain mutually beneficial relationships with similar agents and avoid exploitation by different types of selfish agents.
\end{abstract}

\section{Categories and Subject Descriptors}

I.2.11 [Artificial Intelligence]: Distributed Artificial Intelligence-Multiagent systems

\section{General Terms}

Algorithms, Performance, Economics

\section{Keywords}

negotiation, resources, reciprocity

\section{INTRODUCTION}

We consider multiagent societies where agents repeatedly negotiate with other agents over shared resources and an agent's need and hence preference for a resource varies over time. All negotiations are bilateral and involve one unit of a continuously divisible resource. We assume that negotiating agents have positive utilities for the resource being negotiated and an agent's utility increases with the amount or

Permission to make digital or hard copies of all or part of this work for personal or classroom use is granted without fee provided that copies are not made or distributed for profit or commercial advantage and that copies bear this notice and the full citation on the first page. To copy otherwise, to republish, to post on servers or to redistribute to lists, requires prior specific permission and/or a fee.

AAMAS'07 May 14-18 2007, Honolulu, Hawai'i, USA

Copyright 2007 IFAAMAS share it receives. The utility of a negotiated outcome to an agent is the product of its share of the resource in the outcome and its current valuation for that resource. We assume that an agent does not know the valuation of the other agent for the resource being negotiated. In a single-shot negotiation or negotiations repeated under identical conditions, agents have no incentive to sacrifice any amount of resource at any point of time for the other agents and they end up splitting the resource equally. But, if the agent valuations can change over repeated interactions, which is common in a society, negotiating agents can utilize the corresponding cooperation possibilities. By cooperation possibilities, we refer to situations where one agent requires a limited resource more than another agent [3]. In such a situation, the former agent can benefit more by obtaining a larger than rational share of the resource in this negotiation if the other agent concedes some of its share. The latter agent can similarly ask for such "help" when the preferences are reversed. These cooperative relationships not only benefit individual agents, but can also enhance the performance of the entire society, measured by the sum of utilities of all the agents in the society from all negotiations.

In a real agent society, however, there can be agents who may exploit the helping attitude of the other agents by receiving help without reciprocating. For example, they can lie about their valuations and always state a very high valuation to receive help from the other agents. Our goal is to develop negotiation strategies for repeated interactions such that individual agents cooperate to maximize social welfare but maintain individual competitiveness. Our approach is to use a negotiation protocol that allows agents to form mutually beneficial, trusting relationships where reciprocal cooperation possibilities can be leveraged to maximize both social welfare and individual utilities.

\section{NEGOTIATION IN A SOCIETY}

We consider a society with $N$ agents where each agent repeatedly negotiates shares of resources with other agents. Each negotiation instance involves two agents negotiating over a unit resource. In every time period, each agent is involved in bilateral negotiations with each of the remaining $N-1$ agents. Such agent interactions continue for a negotiation period consisting of $T$ time periods. The value of $T$ is not known to the agents. Later, we use two variations in the societal structure where an agent can negotiate with at most $L$ agents, where $(L<N-1)$, in each time period. In one variation, agents can choose their negotiation part- 
ners. In this scenario, before each negotiation both agents must agree to participate. In the other variation, each agent is randomly matched with $L$ other agents [2] and an agent cannot refuse to participate in any negotiation for which it is selected as a partner.

At any time period $t$, true valuation of an agent $i$ for a resource $j$ is denoted by $d_{i j}^{t}$. The utility obtained by agent $i$ from the negotiation for resource $j$ is $U_{i j}=d_{i j}^{t} * y_{i j}^{t}$, where $y_{i j}^{t} \in[0,1]$ is the share of resource $j$ obtained by agent $i$ after negotiation in the $t^{t h}$ time period.

\section{NEGOTIATION PROTOCOL}

In the proposed negotiation protocol, agents simultaneously communicate before each bilateral negotiation to reveal their valuations for the resource being negotiated. Thereafter, to maximize social welfare, the entire resource is allocated to the agent with the higher valuation. If the revealed valuations are equal, the agents share the resource equally. According to this protocol, if two agents $i$ and $i^{\prime}$ are negotiating over an unit of resource $j$ at time $t$, and $d_{i j}^{*}$ and $d_{i^{\prime} j}^{*}$ are the corresponding stated valuations of agent $i$ and $i^{\prime}$ respectively, agent $i$ receives a share of $y_{i j}^{t}$ given in equation 1 and $y_{i^{\prime} j}^{t}=1-y_{i j}^{t}$, where

$$
\begin{aligned}
y_{i j}^{t} & =1, \text { if } d_{i j}^{t *}>d_{i^{\prime} j}^{t *} \\
& =0, \text { if } d_{i j}^{t *}<d_{i^{\prime} j}^{t *} \\
& =0.5, \text { if } d_{i j}^{t *}=d_{i^{\prime} j}^{t *}
\end{aligned}
$$

Therefore, if all agents truthfully reveal their valuations, this negotiation should lead to maximum social welfare which implies optimal system performance. Equally importantly, this will improve the profit of each individual agent if its valuation is more than its negotiation partner a sufficient number of times. This is because an agent will receive more share of the resources which has higher valuation to it by giving away shares of resources of lower valuation.

Agents, however, have incentive to report high valuations which are more likely to generate higher profits. If an agent always reports highest valuation, a rational agent does not have any incentive to report anything other than the highest valuation when negotiating with that agent. If all agents always report highest valuation $H$, each of the resources will be equally split, which is very inefficient and will reduce social welfare and individual utilities of the negotiating agents. We therefore propose a probabilistic reciprocity based negotiation strategy that utilizes cooperation possibilities in the environment by reporting preferences truthfully when negotiating with other helpful agents ${ }^{1}$ and avoid exploitation by exploitative agents by reporting false high valuations.

\section{AGENT STRATEGIES}

Naive-social agents (NS): Agents who, always reveal their true valuation. This is similar to the pro-social agents [1] who always want to maximize social welfare.

Selfish agents (S): These agents always want to get help from the other agents and never wants to relinquish any share for other agents. They always report the highest valuation, $H$, both to garner help and to avoid giving help. Selfish agents can benefit in the presence of naive-social agents

${ }^{1}$ In this context, when an agent relinquishes its share to another agent with higher valuation, we call it a help given by the first agent to the second one. by exploiting their benevolence.

Reciprocative agents (R): Agents that uses probabilistic reciprocity to decide the valuation it will report. If it decides not to help, it will state the highest valuation $H$, and otherwise state the true valuation and help if a cooperation possibility is found. The valuation reported by agent $i$, when negotiating with agent $j$, depends on $i$ 's previous interactions with agent $j$. If in a negotiation at time $t, i$ relinquishes $x_{i j k}^{t}$ share $^{2}$ of resource $k$ when $i$ 's stated valuation is $d_{i k}^{t}$ and $j$ 's stated valuation is $d_{j k}^{t}\left(d_{j k}^{t}>d_{i k}^{t}\right)$, then $h_{i j}^{t}=x_{i j k}^{t} * d_{j k}^{t}$ is the amount of help agent $i$ has done to agent $j$ at interaction $t$. The total help offered by agent $i$ in all previous interactions with agent $j$ is defined as $C_{i j}=\sum_{t} h_{i j}^{t}$. Similarly, the total help received by agent $i$ from all the previous interactions with agent $j$, denoted by $C_{i j}^{s}$, is defined as $C_{i j}^{s}=C_{j i}$. The difference $\left(C_{i j}^{s}-C_{i j}\right)$ is known as the balance, $B_{i j}$, of agent $i$ from agent $j$.

The probability that agent $i$ will state the true valuation to explore cooperation possibility is given by

$$
\begin{aligned}
P(i, j) & =1, \text { if } B_{i j}>\beta_{i} \text { or } C_{i j}=0 \\
& =0, \text { if } B_{i j}<\alpha_{i} \\
& =\min \left(1, \frac{C_{i j}^{s}}{\gamma * C_{i j}}\right), \text { otherwise. }
\end{aligned}
$$

In Equation $2, \gamma>1$, and $\beta_{i}$ and $\alpha_{i}$ are private parameters of agent $i$ that acts as the upper and lower threshold of the agent's trust in the opponent. If its balance with the opponent agent is more than $\beta_{i}$, it considers that the other agent is completely trustworthy. Similarly, if the balance is less than $\alpha_{i}$ then it considers the other agent as exploitative.

\section{EXPERIMENTAL RESULTS}

In these experiments, all negotiations are bilateral and each negotiation involves one divisible unit of resource. In each negotiation, participating agents' valuation of the resource are two numbers from the set $\{1, \ldots, H\}$. Here we take $H=5$. The valuations for the two negotiating agents are generated before each negotiation using different probability distributions. Let $P(v)$ be the probability that the resource being negotiated has a valuation $v$ to an agent, i.e., $\sum_{v=1}^{H} P(v)=1$. We vary the probability distributions for different experiments to verify the effectiveness of different negotiation behaviors. The total payoff to an agent is the sum of all the utilities it receives from all its negotiations. At the start of a run, each reciprocative $(R)$ agent $i$ chooses its trust bias $\beta_{i}$ from a Gaussian distribution with mean $-H$ and standard deviation 0.5 and $\alpha_{i}$ is taken as $-3 * H$.

We first consider agent societies where each agent participates in a bilateral negotiation with each of the other agents in each time period. We discuss experiments with 90 agents interacting with each other over 200 time periods.

In the first experiment, we have experimented with a mixed group of 45 reciprocative $(R)$ and 45 selfish $(S)$ agents and $P(v)=0.2, \forall v=1, \ldots, 5$. We vary the length of the negotiation period, $T$, and observe its effect on the agents' performance. From the results of this experiment (see Figure 1), we find that when the value of $T$ is less than 35

${ }^{2}$ According to our negotiation protocol, if an agent $i$ has stated a lower valuation than the other agent $j$, agent $j$ gets the entire share of the resource. Agent $i$ relinquishes its half of the entire share. So, $x_{i j k}^{t}=0.5$. 


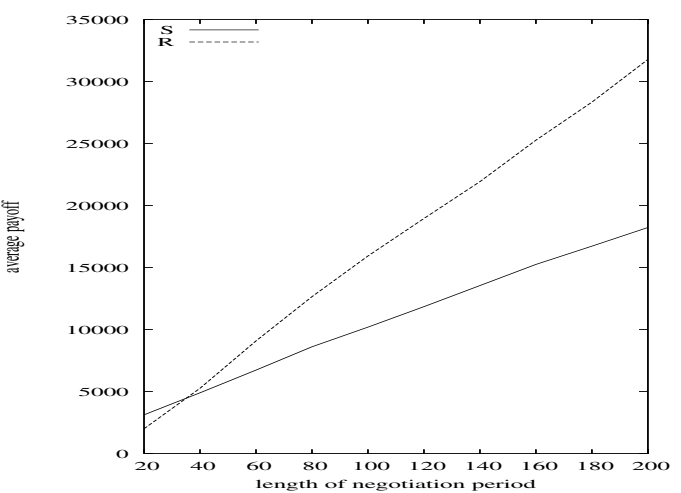

Figure 1: Average payoff of reciprocative and selfish agents for different negotiation periods.

the performance of the selfish $(S)$ agents is better than the performance of the reciprocative $(R)$ agents, but when $T$ is more than $38, R$ agents completely outperform the $S$ agents. When the value of $T$ is between 35 and 38 , the difference in performance is not statistically significant. An $R$ agent initially tries to cooperate in the negotiations by revealing truthfully and giving up its own share when the valuation reported by the other agent is higher. In this period it is exploited by the $S$ agents before it can recognize and stop helping $S$ agents. The $R$ agent cannot recoup these early losses if the length of the negotiation period is small. But when agents are negotiating for a longer period, $R$ agents identify the $S$ agents and stop helping them after some time and thereafter, the initial losses of the $R$ agents can be compensated with the gains obtained from the mutually beneficial negotiations with other $R$ agents.

In the next experiment, we use a mixed group of $N S$, $R$, and $S$ agents. We consider different probability distributions for generating agent valuations that correspond to different extent of cooperation possibilities present in the environment. The agent population is similar to the last experiment. We vary the proportion of $N S$ agents with the rest of the population consisting of equal numbers of $S$ and $R$ agents and compare the performances of the agent behaviors in the mixed group under different conditions. The first situation, $S T 1$, is the situation described in the second experiment, i.e., $P(1)=0.8$, and $P(v)=0.05, \forall v=2, \ldots, 5$. In this situation $S$ agents dominate $R$ agents when more than $30 \%$ agents in the population are NS agents. In the second situation, $S T 2, P(v)=0.2, \forall v=1, \ldots, 5$. With a uniform probability distribution, there are frequent cooperation possibilities in the negotiations as negotiating agents can often have very different resource valuations. From Figure 2, we find that for $S T 2, S$ agents can dominate $R$ agents only when at least $50 \%$ of agents are NS agents. So for $S T 2, S$ agents require much more $N S$ agents, compared to that in $S T 1$, to be present in the environment to dominate $R$ agents. In $S T 2$, the $R$ agents utilize the cooperation possibilities much more than the earlier situation. In the third situation, $S T 3$, we take $P(5)=0.8$ and $P(v)=0.05, \forall v=1, \ldots, 4$. Here, most of the time the valuation of a resource to an agent is 5 and hence cooperation possibilities are infrequent. But as

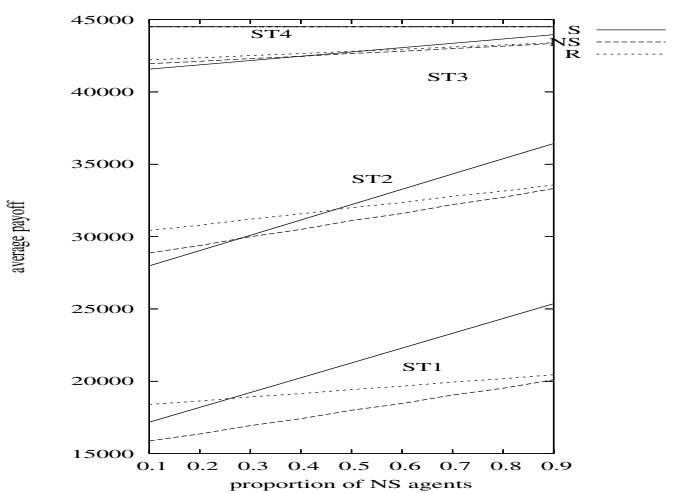

Figure 2: Average payoff of agent types in different negotiation scenarios for varying NS proportions.

the valuation is high, each of the agents earn higher payoff compared to the previous conditions. The fourth situation is an extreme one, where $P(5)=1$. Since each agent has the highest possible valuation for a resource in every negotiation, there are no cooperation possibilities and no help is given and performances of all agents are identical. Note that the average payoff to each agent is maximum here because an agent's valuation is always the highest in this case.

\section{CONCLUSIONS}

We study the evolution of cooperation in repeated negotiation among self-interested agents in a society. We introduce a protocol to maximize social welfare for repeated bilateral negotiation over a shared resource. We then present representative helpful, exploitative, and adaptive strategies for this protocol. Our goal is to identify adaptive strategies that can maximize social welfare and individual utility in environments with sufficient cooperation possibilities. We have designed a robust probabilistic reciprocative strategy that was experimentally shown to form mutually beneficial relationship with similar agents by exchanging help and, at the same time, identify and avoid exploitation by myopic, selfish agents. Hence, rational agents would adopt this strategy which will result simultaneously in social welfare maximization and improvement of individual agent utilities.

Acknowledgment: US National Science Foundation award IIS- 0209208 partially supported this work.

\section{REFERENCES}

[1] C. K. D. Dreu, L. R. Weingart, and S. Kwon. Influence of social motives on integrative negotiations: A meta-analytic review and test of two theories. Journal of Personality and Social Psychology, 78:889-905, 2000.

[2] A. Rubinstein and A. Wolinsky. Decentralized trading, strategic behavior and the walrasian outcome. Review of Economic Studies, 57:63-78, 1990.

[3] S. Sen. Believing others: Pros and cons. Artificial Intelligence, 142(2):179-203, 2002. 